\title{
A Minimal Model of Lung Mechanics and Model based Markers for Optimizing Ventilator Treatment in ARDS Patients
}

\author{
Ashwath Sundaresan ${ }^{1}$, Toshinori Yuta ${ }^{2}$, Christopher E. Hann ${ }^{3}$ \\ J. Geoffrey Chase ${ }^{4}$, Geoffrey M. Shaw ${ }^{5}$, \\ Centre for BioEngineering, Dept of Mechanical Engineering, \\ University of Canterbury \\ Dept of Intensive Care Medicine, Christchurch Hospital \\ Christchurch School of Medicine and Health Sciences, University of Otago \\ Center for BioEngineering \\ Department of Mechanical Engineering \\ University of Canterbury \\ Private Bag 4800 \\ Christchurch \\ New Zealand \\ Correspondence to Ashwath Sundaresan \\ Email: asu33@student.canterbury.ac.nz \\ Telephone: +64 3 364-2987 ext. 7486 \\ Fax: +64 3364-2078
}

\footnotetext{
${ }^{1}$ Research Assistant, Centre for BioEngineering, University of Canterbury

${ }^{2}$ Research Assistant, Centre for BioEngineering, University of Canterbury

${ }^{3}$ Sir Charles Hercus Research Fellow/ Senior Research Associate, Dept of Mechanical Engineering, University of Canterbury

${ }^{4}$ Prof, Dept of Mechanical Engineering, University of Canterbury

${ }^{5}$ Intensive Care Specialist, Dept of Intensive Care, Christchurch Hospital; Sr. Lecturer, Christchurch School of Medicine and Health Sciences, University of Otago
} 


\begin{abstract}
A majority of patients admitted to the Intensive Care Unit (ICU) require some form of respiratory support. In the case of Acute Respiratory Distress Syndrome (ARDS), the patient often requires full intervention from a mechanical ventilator. ARDS is also associated with mortality rate as high as $70 \%$. Despite many recent studies on ventilator treatment of the disease, there are no well established methods to determine the optimal Positive End Expiratory Pressure (PEEP) or other critical ventilator settings for individual patients. A model of fundamental lung mechanics is developed based on capturing the recruitment status of lung units. The main objective of this research is the simplest possible model that is clinically effective in determining PEEP. The model was identified for a variety of different ventilator settings using clinical data. The fitting error was between $0.1 \%$ to $4 \%$ over the inflation limb and between $0.3 \%$ to $13 \%$ over the deflation limb at different PEEP settings. The model produces good correlation with clinical data, and is clinically applicable due to the minimal number of patient specific parameters to identify. The ability to use this identified patient specific model to optimize ventilator management is demonstrated by its ability to predict the patient specific response of PEEP changes before clinically applying them. Predictions of recruited lung volume change with change in PEEP have a median absolute error of 1.87\% (IQR:0.93-4.80\%; 90\% CI:0.16-11.98\%) for inflation and a median of $5.76 \%$ (IQR:2.71-10.50\%; 90\% CI:0.43-17.04\%) for deflation, across all data sets and PEEP values ( $\mathrm{N}=34$ predictions). This minimal model thus provides a clinically useful and simple platform for continuous patient specific monitoring of lung unit recruitment for a patient.
\end{abstract}




\section{Introduction}

A significant number of patients admitted to the ICU require mechanical ventilation due to acute respiratory failure, such as Acute Respiratory Distress Syndrome (ARDS) [1, 2, 3]. The mortality rate of ARDS ranges from around $30 \%$ to as high as $70 \%$ in some studies [4]. In ARDS, the lung is inflamed and filled with fluid, becoming stiff. Furthermore, some lung units collapse from the additional weight of the fluid, reducing the amount of functional lung units. The overall result is a stiffer and volumetrically smaller lung. There are no specific treatments for such acute respiratory diseases, except to facilitate an environment for patients to recover on their own [5].

Mechanical ventilation is used to aid recovery by reducing the work of breathing or taking over this work completely, if necessary. Application of Positive End-Expiratory Pressure (PEEP) is one of the most important intervention in managing a patient with ARDS [6, 7]. PEEP is used to keep the lung partially inflated at the end of expiration in order to prevent lung units from collapsing and maintain their recruitment during subsequent breathing cycles $[8,9]$.

Each patient and their disease state are unique. Thus, the ventilator management needs to be individualized. As the patient's condition changes, the ventilator parameters need to be updated. Specifically, the level of PEEP needs to be adjusted to optimize recruitment and gas exchange, and also to facilitate reductions in mechanical ventilator support, as the patient's condition improves.

Several studies have been conducted to optimize ventilator management, such as low tidal 
volume and PEEP above lower inflection point of the PV curve. These treatments have been shown to reduce mortality in some cases $[7,6]$. However, there are still no well accepted and defined general procedures that can effectively optimize individual ventilator settings over broad cohorts. Hence, clinicians often rely on their own experience and intuition, resulting in widely variable and inconsistent management of mechanical ventilation [10].

This research develops an objective, model-based method of determining the optimal ventilator PEEP treatment using a model estimated level of lung recruitment. The method is based on the simulation and identification of a simplified minimal model of the mechanics of the lung, under a variety of ventilator settings. Some of the mechanics have been examined in earlier stages of this research $[11,12]$. However, there was an excessive number of parameters in earlier models [11] so that it was not uniquely identifiable. Hence, it was not easily applicable for clinical use. This paper describes a minimal model utilizing only two parameters per limb of the breathing curve to track the level of recruitment and its potential use in clinical situations.

\section{Model}

A model of fundamental lung mechanics is developed using simplified physiology and the reported clinical findings. It is based on work by Hickling $[13,14]$, which models the lung as a collection of multiple lung units. A unit represents sets of distal airways and attached alveoli. The lung is divided into several "horizontal" compartments to capture the different levela of superimposed pressure. The compartment at the bottom experiences higher superimposed pressure than the ones above due to the weight of the lung. Figure 1 shows the 
fundamental components of the modelled lung. Any given lung unit has only two possible states at any given pressure: 1) recruited or 2) not recruited.

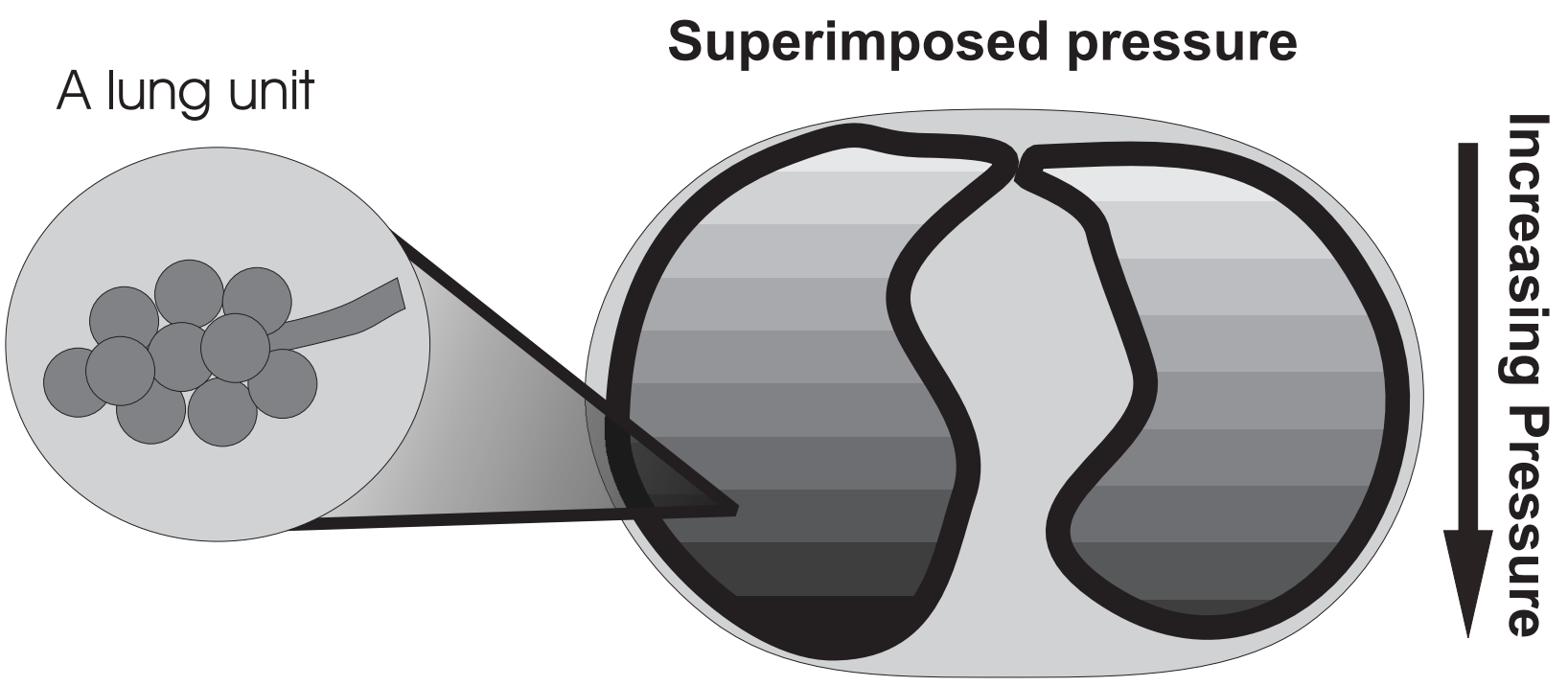

Figure 1: Modelled lung. The lung is modelled as collection of units, evenly divided into compartment of different superimposed pressure.

The mechanism of volume change is based on the hypothesis that it is caused predominantly by recruitment and derecruitment of lung units, especially in ARDS affected lung. Recent in vivo studies have shown that the recruitment and derecruitment of alveoli occurs even in healthy lungs and that once a unit is recruited, it does not change its size significantly $[15,16,17]$. This result suggests that recruitment and derecruitment is the dominant cause of volume change, rather than isotropic "balloon like", expansion of alveoli as had been traditionally thought, which is discussed in [16]. This elastic expansion hypothesis is also utilized by a variety of other clinical studies $[18,19,20,8,13,14,21,22,9,23,24,25,26]$, and is in contrast to the in-vivo and clincally observed, more discrete open-closed behaviour employed here. 


\subsection{Threshold Pressure Distribution}

A modelled lung unit has 2 possible states at a given pressure: 1) Recruited and 2) Derecruited. The recruitment and derecruitment of the modelled lung units are controlled by the distribution of Threshold Opening Pressure (TOP) and Threshold Closing Pressure (TCP), respectively. TOP is the critical pressure at which a previously collapsed unit is recruited during inspriation. Similarly, TCP is the critical pressure where a previously recruited unit collapses during expiration.

Threshold pressures are assumed to be normally distributed along pressure, based on work by Crotti et al. [20] and Pelosi et al. [24]. TOP and TCP distributions can therefore be modelled by a normal density (Gaussian) function. The shape of each distribution is defined by two unique variables: standard deviation (SD) and mean. The total number of lung units in the model are distributed according to these variables within a defined physiological pressure range as well as across superimposed pressure compartments.

Figure 2 shows an example of TOP and TCP distributions. The TOP is distributed broadly from minimum to maximum pressure. This distribution simulates the continuous recruitment of lung units during inspiration. The TCP distribution is significantly narrower compared the TOP, and concentrated at lower pressure. Physiologically, these distributions represent a continuous recruitment of lung units over a full range of pressure during inflation and a more sudden, or faster, derecruitment at lower pressure during deflation. This trend of consistent recruitment through out inspiration and relatively fixed point of derecruitment has been evidenced in several clinical studies [27, 28, 29, 21, 23]. 
The shapes and the values of the distributions are also unique to the condition of the patient and the state of disease. These parameters therefore change as the state of disease and the condition of a given patient evolve. Thus, they can be used as a model based marker to describe the level of recruitment, and thus oxygenation, in the lung and indicate changes in patient condition over time.

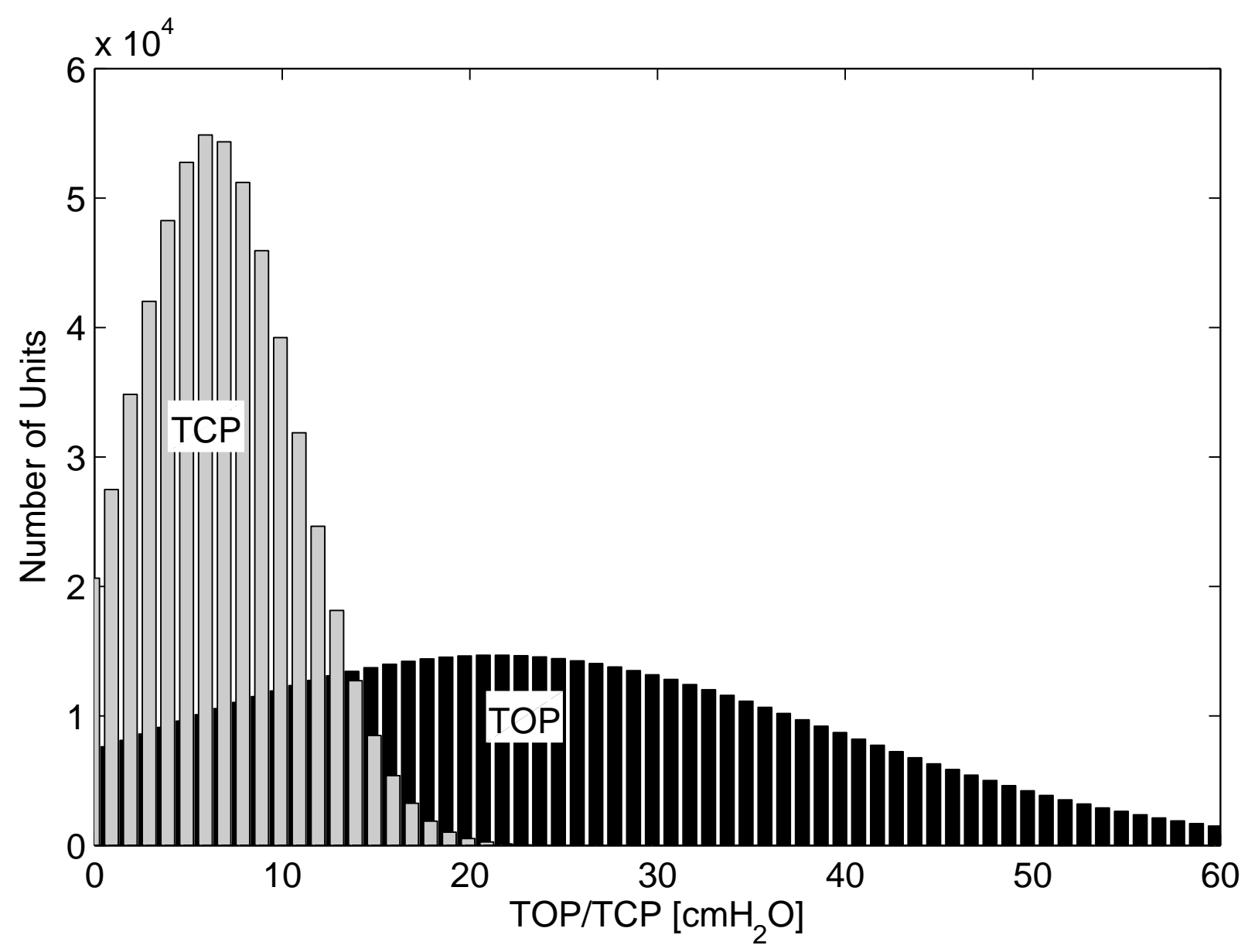

Figure 2: An example of threshold pressure distributions. The distribution is based on normal density function, defined by SD and mean for each distribution. The total number of lung units are distributed within defined pressure range, according to these variables. 


\subsection{Unit Compliance Curve}

Once a lung unit is opened, it assumes a volume defined by a unit compliance curve. The unit compliance is based on a sigmoid curve. An example of a unit compliance curve is shown in Figure 3. The shape and values of the curve is described by the equation:

$$
V=\frac{a}{1+e^{b(-P+c)}}+d
$$

where the volume, $V$, is defined at each pressure increment, $P$, by $a$, the height of the curve, $b$, the curvature, $c$, the midpoint, and $d$, a minimum volume. Equation (1) describes the volume at every pressure value. However, the model only uses a range of specified pressures.

Venegas et al. [30] used a similar equation to describe the entire PV curve. However, in this model, the unit compliance curve only represents visco-elastic effects of recruited alveoli and distal airways. It thus provides the model with slightly more flexibility to match observed clinical behaviour. Since this model is based on the hypothesis that volume change is predominantly caused by recruitment and derecruitment, and that the size of alveoli does not change significantly once recruited, this curve has relatively small effect on the total PV curve. This concept can be seen in the small volume change over a wide range of pressure in Figure 3. Thus, the shape and values for the curve can be fixed during the fitting process, significantly simplifying the analysis and reducing potentially redundant variables. 


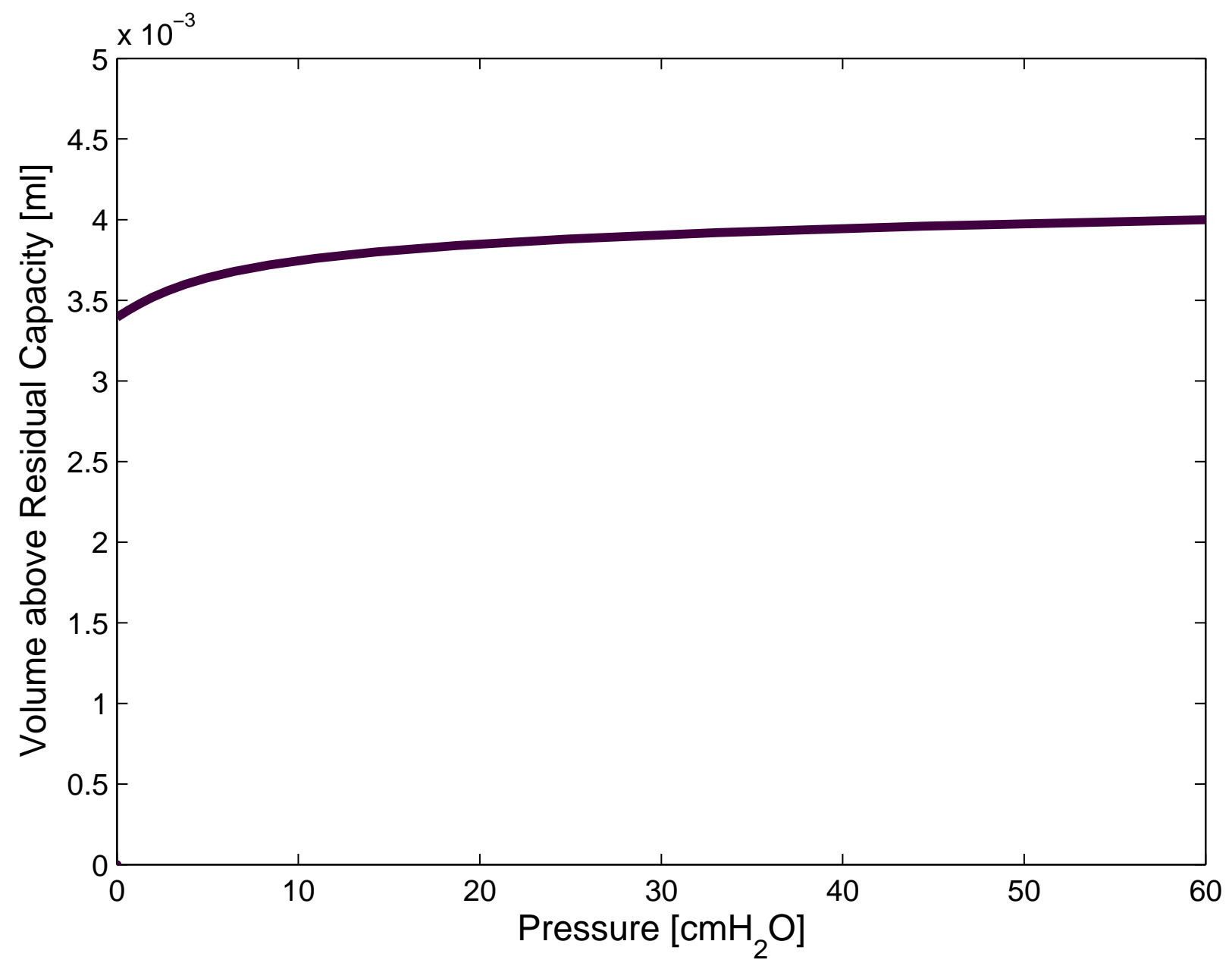

Figure 3: An example of an individual unit compliance curve. The curve is based on a sigmoid function, and defines the volume of a recruited lung unit at a given pressure. The maximum difference in volume is about $20 \%$ in this example.

\subsection{Model Solution/ Simulation}

As pressure changes during a breathing cycle, the model calculates the volume of the lung using the threshold pressure distributions and unit compliance curve. At each pressure in- 
crement, the model evaluates the number of recruited units in each compartment, using appropriate superimposed pressures and threshold pressure distributions. The number of recruited units is then multiplied by the appropriate unit volume according to a unit compliance curve to produce a volume of the compartment. Total lung volume at a specific pressure is the sum of all the compartment volumes. This solution process is shown schematically in Figure 4. Given data from clinical PV loops, the process can be readily inverted to find the threshold pressure distribution parameters. In other words, the model is uniquely and readily identifiable.

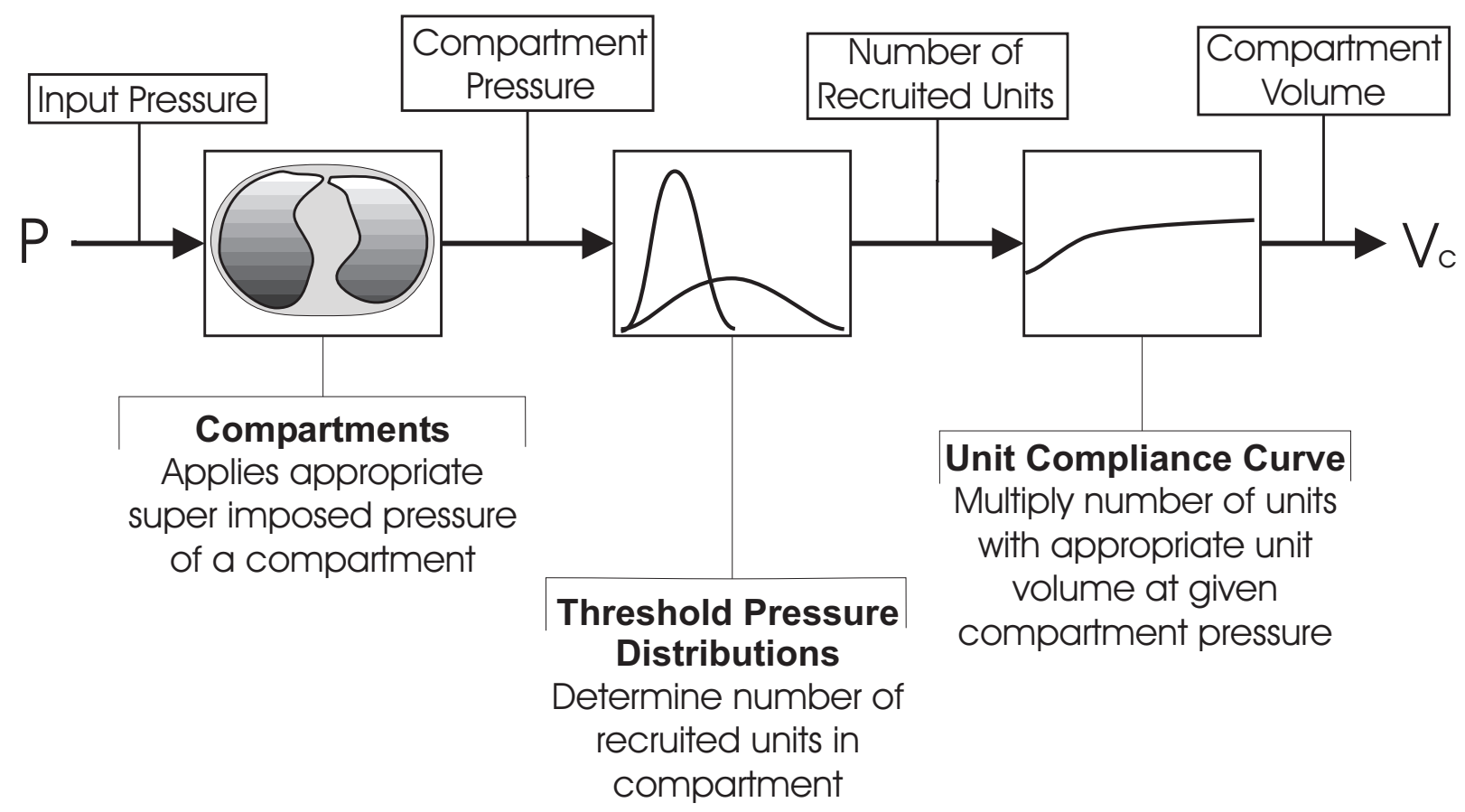

Figure 4: A schematic of the model solution process. It shows each step of the process for calculating one compartment volume. The total lung volume of a given pressure is the sum of all the compartment volumes modelled. 


\subsection{Model Parameter Analysis and Minimization}

A model developed in earlier stages of this research was validated by fitting it to reported clinical data [11]. However, that model included four different types of lung units, based directly on work by Schiller et al. [17], and all different types of unit required unique unit compliance and threshold pressure distributions using results from the literature. The model therefore required as many as 42 patient specific parameters to be identified [11]. Most of those parameters were impractical, if not impossible, to obtain clinically, especially with the limited time, data, and resources in an intensive care unit (ICU). However, the model in [11] was much more physiologically representative. Thus, its relationship to the model presented here is used to define this minimal model's physiological relevance.

The model of [11] was thus modified to create the model presented here, requiring only 2 parameters per breathing limb to make it clinically applicable. First, reducing the number of unit types to just one, reduced the number of parameters to 14. Second, using the hypothesis that most of the volume change is caused by recruitment and derecruitment [e.g. 15, 16, 17], the unit compliance has a relatively smaller contribution to the overall PV curve shape. As a result, the unit compliance curve defined in Equation 1 can be fixed at generic values. Finally, fixing the maximum and minimum threshold pressures to 60 and $0 \mathrm{cmH}_{2} \mathrm{O}$, respectively, to cover the range of typically used ventilation pressures, the total number of parameters was reduced to just four:

- TOP distribution mean - Inspiratory limb of breathing curve

- TCP distribution mean - Expiratory limb of breathing curve 
- TOP standard deviation - Inspiratory limb of breathing curve

- TCP standard deviation - Expiratory limb of breathing curve

These parameters are effectively two for the inflation and deflation limbs. Other variables, such as PEEP, PIP, and tidal volume are assumed known, as they are set by the clinician or can be obtained directly from ventilator.

Hence, the changes made do not remove any significant physiological representation. What is lost is the level of physiological detail in the number and the types of ARDS affected and healthy lung units. However, these values were not uniquely identifiable without as many as 20 unique PV curves, which was not clinically practical.

In their place is now a single unit with the two states of recruited and derecruited. However, these two states can, at a given pressure, represent the level of ARDS by the level of recruitment. More specifically, as ARDS progresses, there is less recruitment at a given pressure and PEEP, which is effectively captured by the four (2 each) parameters describing TOP and TCP.

As a result, the parameter identification is greatly simplified and importantly, is unique, given a reasonably discretized measured PV curve. The main requirement is a minimum of 2 complete PV loops to provide enough data to identify the two parameters for the inflation, TOP distribution, and deflation, TCP distribution, limbs. A second requirement is that these loops be obtained at clincally different PEEP values. TOP and TOP parameter identification is readily done by iteratively modifying the threshold pressure distribution 
variables to minimize the sum squared error between the model and clinical data for each limb of the PV loops.

Since the inflation and deflation limbs are generated by different independent parameters, each limb can be fitted separately. The PEEP value sets the minimum pressure for the PEEP to PIP breathing cycle. The standard deviation of the TOP or TCP distribution primarily controls the slope of the curve and the mean value primarily controls the location along the pressure axis of the respective curves.

\section{Model Validation}

The model was validated by fitting to clinical PV data at different PEEP levels reported in the literature [31]. The study used data from 10 patients each with a different level of lung injury. Each data set had at least three different PEEP levels with the associated deflation to the Functional Residual Capacity (FRC) having to be measured by intervention. Using this measurement, it is then possible to evaluate the End Expiratory Volume (EEV) for a given PEEP.

For this validation analysis, data at different PEEP settings from the same patient are fitted by shifting the distribution mean value, while other parameters were fixed. This shift represents the effect of the dynamic mechanism of lung units at different PEEP values. More specifically, once a collapsed lung unit is recruited, it does not necessarily collapse again at the same pressure at which it was recruited. Instead, it stays recruited at a lower pressure [21]. This effect is especially significant in the ARDS lung because of the reduced number of 
functional lung units and lower compliance of the overall lung. The benefit of recruitment manoeuvres on ventilated patients is based on this dynamic $[32,33]$. The same effect results from increasing the PEEP, while keeping the tidal volume the same, causing higher Peak Inspiratory Pressure (PIP).

This behaviour results in a shift in threshold pressure distributions in the model. Therefore, as PEEP is increased, the center of the TOP distribution shifts to lower pressure, indicating that more units are kept recruited during the breathing cycle. Hence, they begin to recruit, and keep recruited, lung units that were collapsed at lower PEEP settings. Again, this physiological dynamic has been observed in several clinical studies [e.g. 32, 8, 22, 9, 33].

Similarly, the TCP distribution mean shifts to higher pressure indicating unit instability at relatively higher pressures compared to TCP at lower PEEP settings. The higher PEEP recruits more units in regions with higher superimposed pressure and injured units. These newly recruited units are inherently unstable, and thus, are likely to be de-recruited at relatively higher pressure. Hence, the higher PEEP increases the recruitment of previously collapsed units at a given pressure, increasing the number of functional units. However, those units are unstable and collapse at relatively higher pressure.

The amount of the shift in both distributions is dependent on the individual patients' condition and state of disease. More specifically, PEEP is modified to increase recruitment and optimize oxygenation. Therefore, tracking the number of recruited units at a given pressure and PEEP over time is a measure of recruitment that effectively tracks the state of ARDS or lung disease using the level of recruitment. Thus, it can be used as a key parameter to describe the characteristics of a patient specific lung, track the level of recruitment, and 
quantitatively determine the impact of therapy.

The method for fitting the data is based on simulating the entire inspiratory capacity of the lung. For a given recruitment status of the lung, Figure 5 shows an example of a typical PV curve as obtained from either standard Volume Controlled Ventilation (VCV) or Pressure Controlled Ventilation (PCV) [31]. Also shown is the tidal volume, PEEP and PIP.

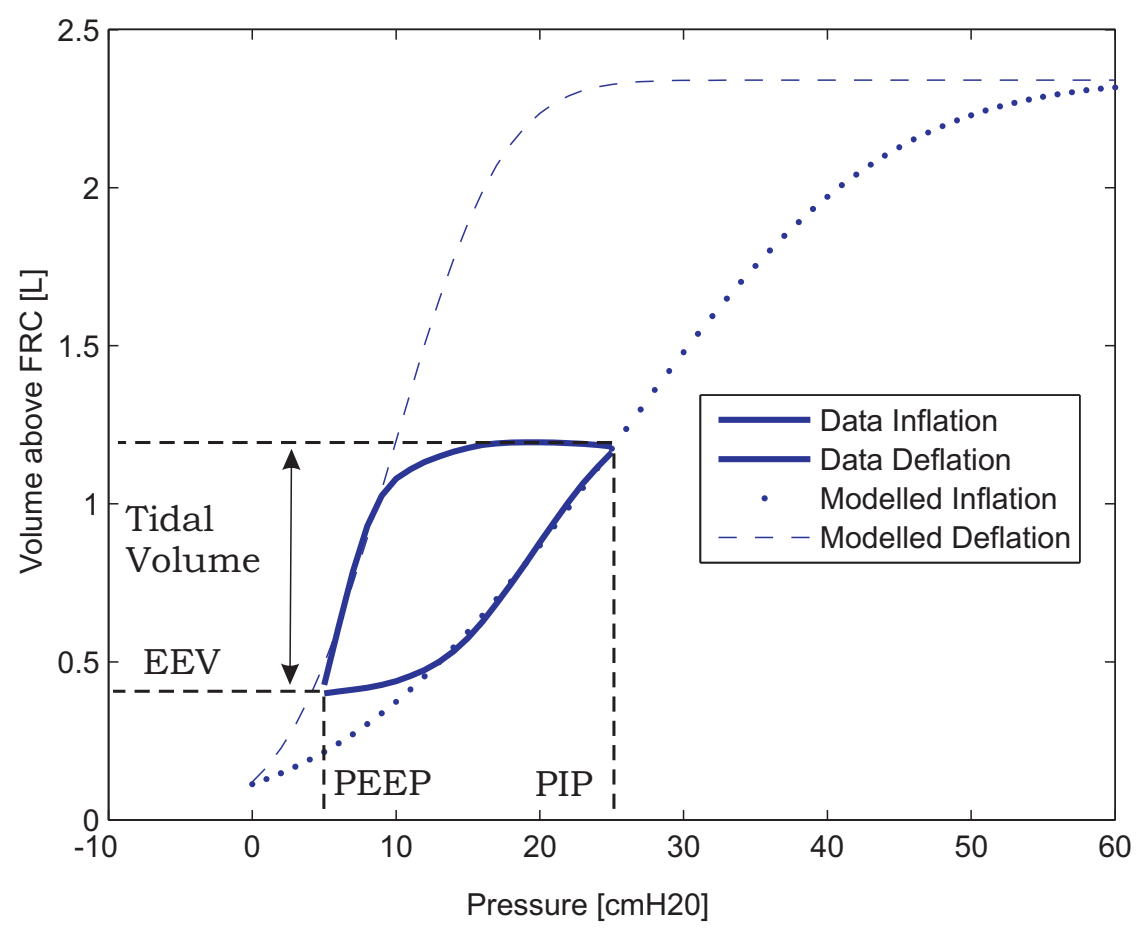

Figure 5: An example of the model fit. The plot shows the modelled inflation, modelled deflation and the data for inflation and deflation. The model fits the entire lung capacity.

The lower dotted curve represents the inflation curve which is dependent on the type of ventilation used. The upper dashed curve represents the quasi-static PV curve which is ventilator independent and represents the steady state volume obtainable for each pressure increment 
from 0 to $60 \mathrm{cmH}_{2} \mathrm{O}$. Again, note that the figure is only valid for a specific recruitment status of the lung. In practice, the alveoli recruitment dynamics can change if different settings of PEEP are used for sufficiently long periods. The result of this physiological effect would be a change in the curves of Figure 5, and a shift in the means of the corresponding TOP and TCP distributions.

The upper dashed curve also represents the limiting behaviour of the deflation part of the PV relationship for the given recruitment status, and in principle could be obtained experimentally by a sequence of pressure holds from 0 to $60 \mathrm{cmH}_{2} \mathrm{O}$.

For the example in Figure 5 the limiting behaviour is constrained by the given PEEP value. In other words, during the passive expiratory phase of $\mathrm{VCV}$, the pressure drops very quickly and stays close to PEEP, so that the volume must eventually settle onto the quasi-static PV curve. The last part of this deflation curve is therefore effectively equivalent to a pressure hold at PEEP.

Another way of describing the upper expiratory dashed curve is that it is the asymptotic behaviour of the deflation curve from PIP to PEEP. Similarly, the lower dotted line represents the asymptotic behaviour of the inflation period.

Hence, at the start of inflation and end of deflation there is a transient period where the PV curve is seeking to settle on the global asymptotic curves shown in Figure 5 . This period does represent real lung mechanics but does not significantly reflect the volume responsiveness described by EEV and PIP, which are the most important variables, clinically. The transient behaviour is also evident in the PV data of Figure 6, where the sparse point distributions indicate this rapid transition, resulting in a reduced amount of sampled data. 


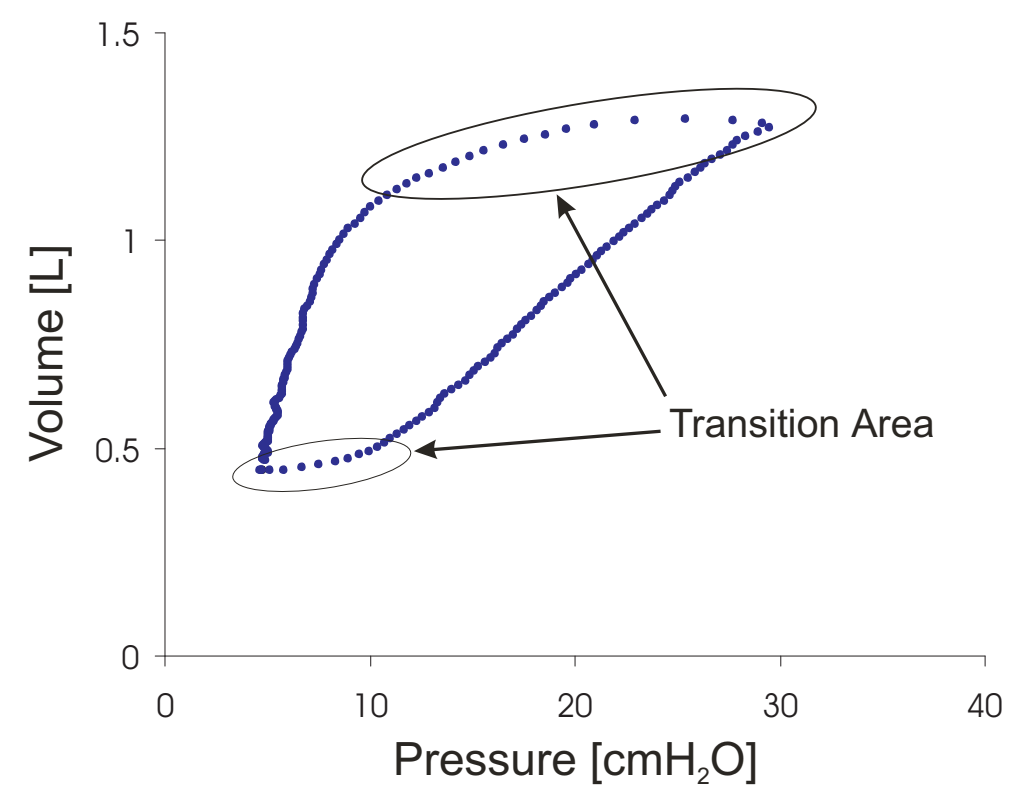

Figure 6: PV Loop showing the transient behaviour during inflation and deflation.

Figures 5 and 6 thus motivate a fitting procedure that uses the last approximately $60 \%$ of the inflation curve and the densely sampled $70 \%$ (by volume) of the deflation curve. These numbers were chosen experimentally and are essentially arbitrary, but cannot be too large otherwise the less sampled, transient periods would induce an error. Alternatively, they cannot be too small or the lack of data would cause unidentifiablity in the model.

Figures 7 and 8 show the model fit for two clinical PV data sets from [31]. The dotted line shows the clinical data and the solid line shows the model fit across the densely sampled non-transition region. The model was fitted to all the PV loops shown. However, only two model fits per data set are shown here for clarity.

The identification and fitting error was calculated using the percentage difference between 


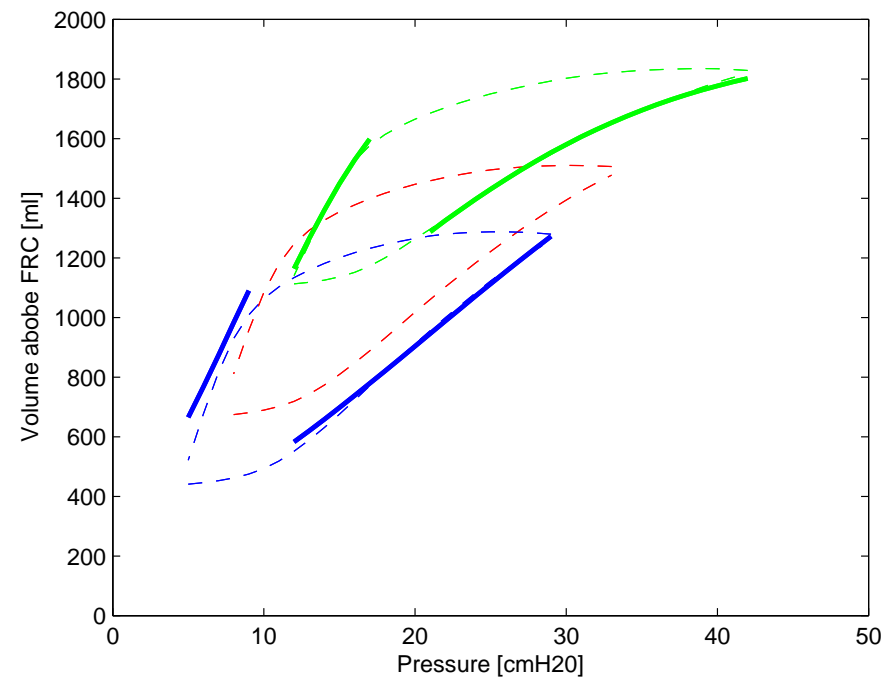

Figure 7: Data Set 1. Dotted line shows the clinical data and solid line shows the model fitted to the clinical data [31]. Model fits for PEEP $=5$ and $12 \mathrm{cmH}_{2} \mathrm{O}$ are shown, with $\mathrm{PEEP}=7 \mathrm{cmH}_{2} \mathrm{O}$ not fitted.

the model and the data in the inflation and deflation limb for each PV loop data set. These results are shown in Table 1. Overall, the loops in Figure 7 show little significant deviation and a good match to the measured data. Similar results are observed in Figure 8. Table 1 shows these results are relatively tight.

\begin{tabular}{|l|c|c|c|c|c|}
\hline & \multicolumn{5}{|c|}{ PEEP $\left[\mathbf{c m H}_{2} \mathbf{O}\right]$} \\
\hline \multirow{3}{*}{ Data Set 1 } & & 5 & 7 & 10 & \\
\cline { 2 - 6 } & Inflation & $1.71 \%$ & $0.48 \%$ & $0.34 \%$ & \\
& Deflation & $12.33 \%$ & $4.25 \%$ & $0.92 \%$ & \\
\hline \multirow{3}{*}{ Data Set 2 } & & 5 & 7 & 10 & \\
\cline { 2 - 6 } & Inflation & $1.74 \%$ & $0.53 \%$ & $0.59 \%$ & \\
& Deflation & $3.99 \%$ & $2.59 \%$ & $2.36 \%$ & \\
\hline \multirow{3}{*}{ Data Set 3 } & & 5 & 7 & 10 & 12 \\
\cline { 2 - 6 } & Inflation & $3.03 \%$ & $0.79 \%$ & $0.58 \%$ & $0.63 \%$ \\
& Deflation & $10.02 \%$ & $7.49 \%$ & $5.57 \%$ & $3.09 \%$ \\
\hline
\end{tabular}

Continued on Next Page... 


\begin{tabular}{|c|c|c|c|c|c|}
\hline & & PEE & {$\left[\mathbf{c m H}_{2}\right.$} & & \\
\hline & & 5 & 7 & 10 & 12 \\
\hline Data Set 4 & Inflation & $3.47 \%$ & $1.24 \%$ & $1.03 \%$ & $0.48 \%$ \\
\hline & Deflation & $9.83 \%$ & $2.48 \%$ & $1.12 \%$ & $0.75 \%$ \\
\hline & & 5 & 7 & 10 & 12 \\
\hline Data Set 5 & Inflation & $1.86 \%$ & $0.58 \%$ & $0.40 \%$ & $0.33 \%$ \\
\hline & Deflation & $7.09 \%$ & $2.87 \%$ & $0.53 \%$ & $0.24 \%$ \\
\hline & & 5 & 7 & 12 & \\
\hline Data Set 6 & Inflation & $2.50 \%$ & $1.16 \%$ & $0.85 \%$ & \\
\hline & Deflation & $5.15 \%$ & $1.86 \%$ & $1.02 \%$ & \\
\hline & & 10 & 12 & 15 & \\
\hline Data Set 7 & Inflation & $0.78 \%$ & $0.47 \%$ & $1.01 \%$ & \\
\hline & Deflation & $1.19 \%$ & $0.78 \%$ & $0.82 \%$ & \\
\hline & & 5 & 7 & 10 & 12 \\
\hline Data Set 8 & Inflation & $2.59 \%$ & $0.54 \%$ & $0.05 \%$ & $0.54 \%$ \\
\hline & Deflation & $9.39 \%$ & $4.04 \%$ & $2.19 \%$ & $1.07 \%$ \\
\hline & & 5 & 7 & 10 & \\
\hline Data Set 9 & Inflation & $1.62 \%$ & $1.24 \%$ & $0.62 \%$ & \\
\hline & Deflation & $10.59 \%$ & $5.34 \%$ & $2.48 \%$ & \\
\hline & & 5 & 10 & 15 & \\
\hline Data Set 10 & Inflation & $2.30 \%$ & $3.60 \%$ & $0.93 \%$ & \\
\hline & Deflation & $8.05 \%$ & $5.17 \%$ & $1.57 \%$ & \\
\hline
\end{tabular}

Table 1: Summary of model errors

\section{Clinical Application: Model Prediction}

The main objective of this research is to develop the simplest possible model that is also clinically useful in selecting a proper PEEP value. One approach is to provide an objective method of determining optimal ventilator settings or PEEP for an individual patient. An- 


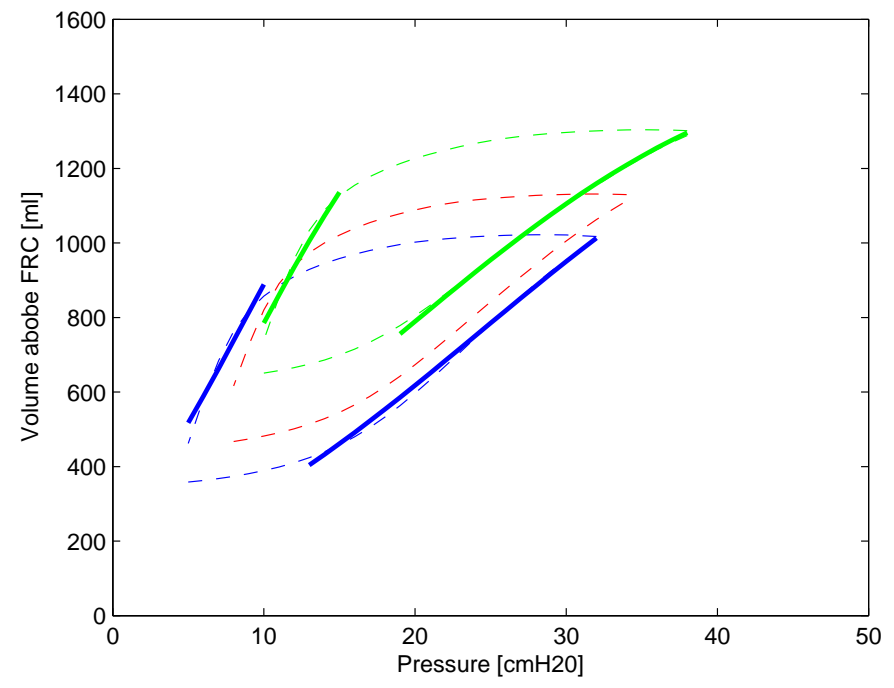

Figure 8: Data Set 2. Dotted line shows the clinical data and solid line shows the model fitted to the clinical data [31]. The model is fit for $\mathrm{PEEP}=5$ and $10 \mathrm{cmH}_{2} \mathrm{O}$, as shown, with the intermediate $\mathrm{PEEP}=7 \mathrm{cmH}_{2} \mathrm{O}$ not fitted.

other is to provide constant monitoring for a patient's level of lung recruitment, and thus the level of ARDS and the impact of therapy as patient condition evolves.

The overall average pressure point error for Data Set 1 was $14.3 \mathrm{ml}(1.40 \%)$ and $60.35 \mathrm{ml}$ $(6.36 \%)$, for inflation and deflation respectively. For Data Set 5, the average absolute error was $15.3 \mathrm{ml}(1.89 \%)$ and $40.5 \mathrm{ml}(6.36 \%)$, for inflation and deflation respectively. Table 2 summarises the prediction errors for these 2 data sets for each predicted PEEP value.

\begin{tabular}{|l|c|c|c|c|c|c|}
\hline & & & \multicolumn{4}{|c|}{ PEEP $\left[\mathbf{c m H}_{2} \mathbf{O}\right]$} \\
\cline { 3 - 7 } Data Set 1 & & 5 & 7 & 10 & \\
\cline { 2 - 7 } & Inflation & Error[mls] & 16.13 & 9.98 & 16.83 & \\
& & Error[\%] & 2.23 & 0.89 & 1.10 & \\
& \multirow{2}{*}{ Deflation } & Error[mls] & 49.26 & 67.16 & 64.65 & \\
& & Error[\%] & 7.58 & 6.71 & 4.78 & \\
\hline
\end{tabular}

Continued on Next Page... 


\begin{tabular}{|c|c|c|c|c|c|c|}
\hline & & & & EEP & $\left.\mathrm{mH}_{2} \mathrm{O}\right]$ & \\
\hline & & & 5 & 7 & 10 & \\
\hline Data Set 2 & Inflation & Error $[\mathrm{mls}]$ & 14.02 & 10.99 & 24.87 & \\
\hline & & Error[\%] & 2.09 & 1.33 & 2.60 & \\
\hline & Deflation & Error $[\mathrm{mls}]$ & 82.18 & 54.24 & 133.59 & \\
\hline & & Error[\%] & 11.69 & 6.80 & 13.92 & \\
\hline & & & 5 & 77 & 10 & 12 \\
\hline Data Set 3 & Inflation & Error $|\mathrm{mls}|$ & 15.86 & 10.18 & 16.38 & 18.91 \\
\hline & & Error $[\%]$ & 2.35 & 1.48 & 1.97 & 1.75 \\
\hline & Deflation & Error $[\mathrm{mls}]$ & 15.61 & 88.64 & 15.74 & 42.08 \\
\hline & & Error $[\%]$ & 3.04 & 14.91 & 2.61 & 4.90 \\
\hline & & & 5 & 7 & 10 & 12 \\
\hline Data Set 4 & Inflation & Error $[\mathrm{mls}]$ & 38.17 & 41.10 & 29.81 & 14.38 \\
\hline & & Error $[\%]$ & 8.21 & 7.41 & 4.02 & 1.56 \\
\hline & Deflation & Error $[\mathrm{mls}]$ & 48.50 & 72.87 & 32.76 & 5.41 \\
\hline & & Error $[\%]$ & 13.44 & 16.97 & 4.64 & 0.73 \\
\hline & & & 5 & 7 & 10 & 12 \\
\hline Data Set 5 & Inflation & Error $[\mathrm{mls}]$ & 31.87 & 35.09 & 21.82 & 10.63 \\
\hline & & Error $[\%]$ & 2.52 & 2.33 & 1.11 & 0.48 \\
\hline & Deflation & Error $[\mathrm{mls}]$ & 35.60 & 112.82 & 13.33 & 9.06 \\
\hline & & Error $[\%]$ & 3.32 & 8.89 & 0.72 & 0.40 \\
\hline & & & 5 & 7 & 12 & \\
\hline Data Set 6 & Inflation & Error $|\mathrm{mls}|$ & 134.87 & 107.40 & 268.82 & \\
\hline & & Error $[\%]$ & 19.00 & 10.37 & 16.34 & \\
\hline & Deflation & Error $[\mathrm{mls}]$ & 70.30 & 53.75 & 153.21 & \\
\hline & & Error $[\%]$ & 11.22 & 4.92 & 9.26 & \\
\hline & & & 10 & 12 & 15 & \\
\hline Data Set 7 & Inflation & Error $[\mathrm{mls}]$ & 97.25 & 48.48 & 101.40 & \\
\hline & & Error $[\%]$ & 7.17 & 2.82 & 5.10 & \\
\hline & Deflation & Error [mls] & 137.55 & 75.43 & 183.81 & \\
\hline & & Error $[\%]$ & 11.20 & 4.95 & 10.64 & \\
\hline & & & 5 & 7 & 10 & 12 \\
\hline Data Set 8 & Inflation & Error $[\mathrm{mls}]$ & 19.79 & 10.69 & 8.05 & 8.15 \\
\hline & & Error[\%] & 2.89 & 0.88 & 0.58 & 0.47 \\
\hline & Deflation & Error [mls] & 47.49 & 54.15 & 19.91 & 18.86 \\
\hline & & Error $[\%]$ & 7.35 & 5.43 & 1.78 & 1.43 \\
\hline & & & 5 & 7 & 10 & \\
\hline Data Set 9 & Inflation & Error [mls] & 15.29 & 16.39 & 14.69 & \\
\hline & & Error $[\%]$ & 1.69 & 1.29 & 0.84 & \\
\hline & Deflation & Error $[\mathrm{mls}]$ & 17.00 & 111.24 & 101.43 & \\
\hline & & Error $[\%]$ & 2.23 & 10.48 & 7.06 & \\
\hline
\end{tabular}

Continued on Next Page... 


\begin{tabular}{|c|c|c|c|c|c|}
\hline & & & \multicolumn{3}{|c|}{ PEEP $\left[\mathrm{cmH}_{2} \mathrm{O}\right]$} \\
\hline & & & 5 & 10 & 15 \\
\hline \multirow[t]{4}{*}{ Data Set 10} & Inflation & Error $[\mathrm{mls}]$ & 16.43 & 26.79 & 19.13 \\
\hline & & Error $[\%]$ & 4.51 & 4.24 & 1.83 \\
\hline & Deflation & Error $[\mathrm{mls}]$ & 43.58 & 40.81 & 38.80 \\
\hline & & Error $[\%]^{\lrcorner}$ & 9.65 & 7.79 & 4.09 \\
\hline
\end{tabular}

Table 2: Summary of PV prediction errors for all data

sets

Table 3 summarises the overall prediction for all 10 data sets. This table shows the maximum and average percentage absolute error for inflation and deflation at each predicted PEEP level. The interquartile range of errors are shown to provide the range of errors.

\begin{tabular}{|l|c|c|c|c|c|c|c|c|}
\hline & \multicolumn{4}{|c|}{ Inflation } & \multicolumn{4}{c|}{ Deflation } \\
\cline { 2 - 9 } PEEP $\left[\mathrm{cmH}_{2} \mathrm{O}\right]$ & Avg & IQR & $90 \%$ CI & Max & Avg & IQR & $90 \%$ CI & Max \\
\hline 5 & 5.04 & $1.07-7.39$ & $0.14-18.02$ & 27.54 & 8.19 & $2.81-12.42$ & $0.88-20.84$ & 27.98 \\
7 & 3.15 & $0.93-4.15$ & $0.20-9.84$ & 19.20 & 9.42 & $5.57-12.53$ & $2.86-22.37$ & 26.87 \\
10 & 2.89 & $0.91-4.16$ & $0.30-8.73$ & 13.55 & 6.44 & $1.42-9.72$ & $0.27-16.11$ & 22.08 \\
12 & 3.32 & $0.69-2.69$ & $0.03-19.53$ & 22.85 & 3.84 & $0.89-5.57$ & $0.03-9.44$ & 14.16 \\
15 & 3.62 & $1.99-5.10$ & $1.10-5.82$ & 6.25 & 7.67 & $5.03-11.03$ & $1.49-11.67$ & 11.68 \\
\hline
\end{tabular}

Table 3: Summary of PV prediction percentage errors for all data sets. The errors are listed according to predicted PEEP levels

The data that the model requires for both uses can be directly obtained from the ventilator during normal operation and clinical adjustment. All the analysis can be done at bedside and be immediately applied. Furthermore, it does not require additional equipment or tests. Thus, additional cost and risk to the patient are avoided. 
The ventilator setting optimization procedure is done in 3 steps: 1) parameter identification, 2) simulation of a change in PEEP, and 3) analysis. The model was validated by using clinical data from Bersten [31]. The clinical data in this study were recorded at different PEEP settings for each patient and each measurement included an EEV measurement. The data was sampled for 60 seconds at $100 \mathrm{~Hz}$ for each PEEP level PV loop using volume controlled ventilation. The data also included a deflation to FRC and the last 15 to 20 seconds were used for the FRC measurement.

Because the model can identify lung mechanics over an entire pressure range, it can be used to predict the lung mechanics at different ventilator settings. Thus, the model allows the clinicians to see the result before it is actually applied to the patient, and decide on whether to apply the changes. The model also allows predictions about multiple results to be made quickly. Quick turnaround eliminates the need for clinical trial and error procedures. Hence, optimum PEEP and ventilator settings can be obtained via simulation if the model predictions are accurate.

Therefore, for patients with data at 3 PEEP settings, 2 PEEP settings are used to fit the model and the mean shift trend. For example, Figure 9 shows the parameter identification for two measured clinical PV loops. The plot shows the clinical PV loops with the fitted model results and the respective threshold distributions. The fitted model is then tested to predict the results of the remaining PV loop. If 4 PEEP settings are available, then the model is fitted with 3 loops. Any combination can be used to test the remaining unused PEEP value and PV curve.

Specifically, if 2 PV loops are obtained at higher PEEP values, then the model can predict 


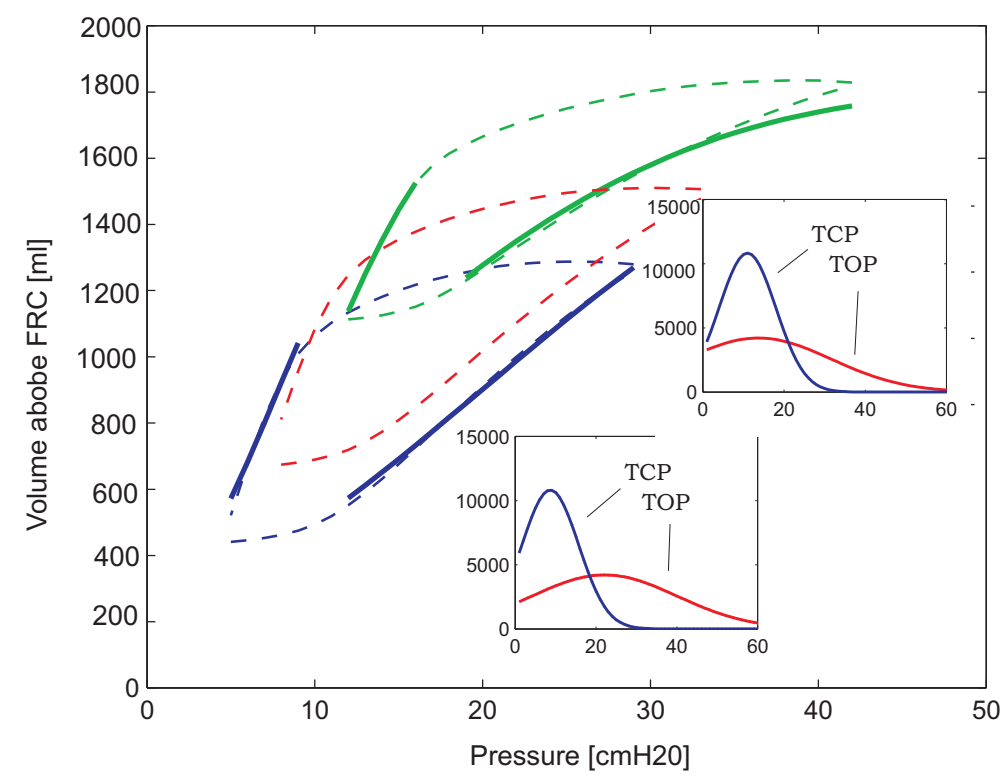

Figure 9: Data fitting for parameter identification. The essential parameters are identified by fitting the model to the clinical data. Each loop results in unique threshold pressure distributions defining level of recruitment for that PEEP. Respective threshold pressure distributions show the mean of TOP has moved left with higher PEEP setting and the TCP mean has moved right.

a PV loop for a lower PEEP. Prediction of a lower PEEP is useful in the clinical setting as it means that alveolar collapse can be avoided as a result of PEEP reduction. Similarly, predicting higher PEEP level responses allows evaluation of the clinical impact in recruitment of changing PEEP.

The model was able to predict the missing mean shift and PV curve for the PEEP level with relatively small errors. The error metric chosen is average relative percentage error, computed over the densely sampled portions of the PV loops used in the identification process. This 
approach avoids the transient period, as discussed earlier in Figures 5 and 6

Figure 10 illustrates the fitted mean shift and actual mean for PEEP of $5 \mathrm{~cm}_{2} 0$ in Data Set 1 as predicted, having fitted for data for PEEP $=7$ and $12 \mathrm{cmH}_{2} 0$. The predicted value is marked with an asterisk $\left(^{*}\right)$. Figure 11 shows the resulting PV curve prediction, where the dashed lines are the original clinical data for the predicted PV loop. Because the model was not fit in the highly dynamic transition area, the prediction was also made only for the steady portion of the curve, as illustrated by the dots in the figure.

Figures 12 and 13 illustrates the same prediction result for PEEP levels for 7 and $12 \mathrm{cmH}_{2} 0$ for Data Set 1, respectively. All results show minimum errors that are clinically insignificant. Finally, note that the linear mean shift trend lines in Figures 10 to 13 are all effectively identical with minimal difference.

Figures 14 to 17 illustrate the same results for all PEEP level predictions in the larger Data Set 5. In contrast to Data Set 1, the slope of these patient specific mean shift values is lower, indicating a patient thats much less responsive to changes in PEEP. More specifically, this patient experiences far less of an increase in recruited lung volume for a given increase in PEEP, indicating less clinical effect in modifying therapy. Similar to the results for Data Set 1, all the predicted PV curves show clinically insignificant levels of error in their predictions based on mean shift.

The results of this model show that as PEEP increases, the TOP distribution mean decreases, while the TCP distribution mean increases. These shifts indicate the change in recruitment and derecruitment characteristics of lung units as PEEP is altered $[8,22,9]$. 


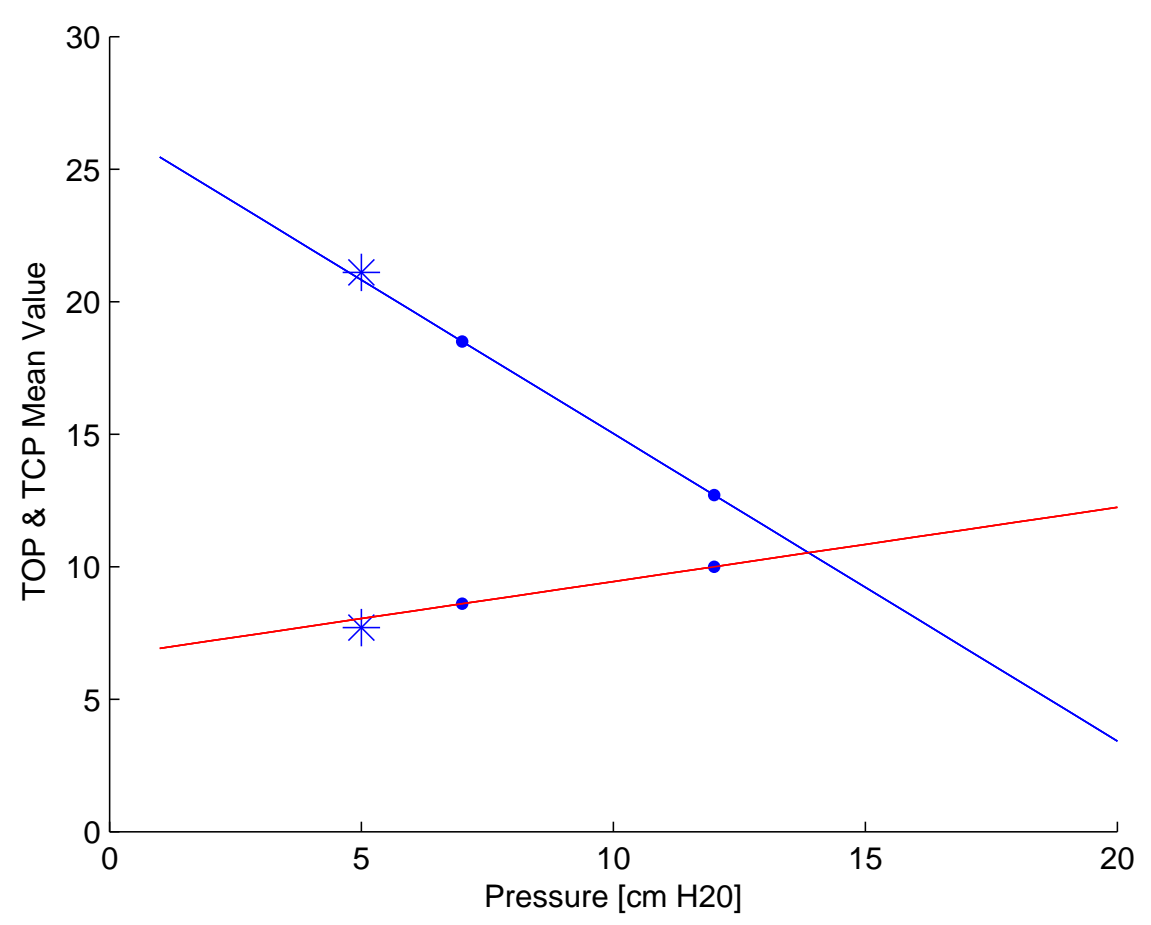

Figure 10: A fitted mean shift for prediction result for Data Set 1. PEEP $=7 \mathrm{cmH}_{2} 0$ and $\mathrm{PEEP}=12 \mathrm{cmH}_{2} 0$ were used to predict PV data at PEEP $=5 \mathrm{cmH}_{2} 0$. The linear lines for mean shift (dashed lines) are identified from the 2 given data sets (solid dots). The ${ }^{*}$ shows the mean being predicted. Red and blue lines are for the TCP and TOP distribution mean, respectively.

The model requires a minimum of two PV loops at different PEEP settings to obtain all the required parameters. However, more data will increase the accuracy of the parameters and resulting simulation, particularly in presence of noise. The values of PEEP used do not need to be predetermined or specified, but should be within a safe range determined by medical staff for that specific patient and be reasonably different. Once a best fit is achieved, the model records those parameter values for prediction and simulation. 


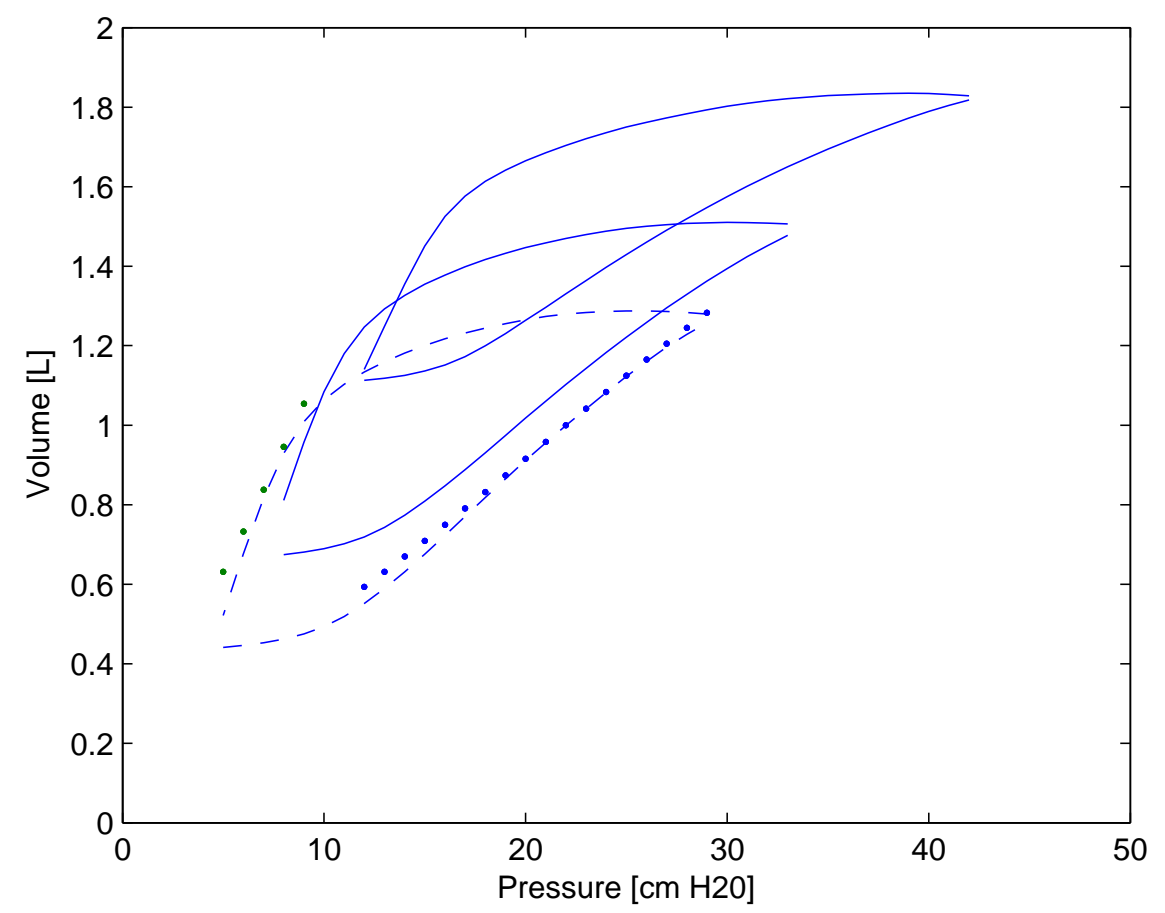

Figure 11: A prediction result for PEEP $=5 \mathrm{cmH}_{2} \mathrm{O}$ of Dataset 51. The PV data was predicted by fitting 2 known PEEP levels (solid lines). The green and blue dots show the predicted data and dashed lines show original lines.

The model can simulate the lung for any combination of PEEP and tidal volume, including the extreme values that may not be safe to test on an actual patient. Since the model is based on a reduced number of parameters and simplified mechanics, the simulation can be done in matter of seconds. This produces real-time feedback and a condition specific result.

The volume shifts between different means are captured by the shifting distribution means. In general, the TOP mean decreased as PEEP increased, and the TCP mean increased as PEEP increased. Physiologically, this indicates the varying nature of TOP an TCP under 

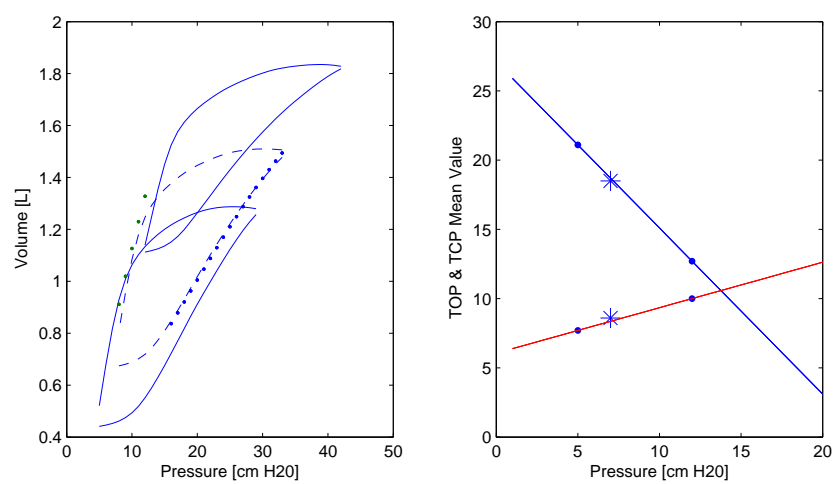

Figure 12: A prediction result for $\mathrm{PEEP}=7 \mathrm{cmH}_{2} \mathrm{O}$ of Dataset 1.
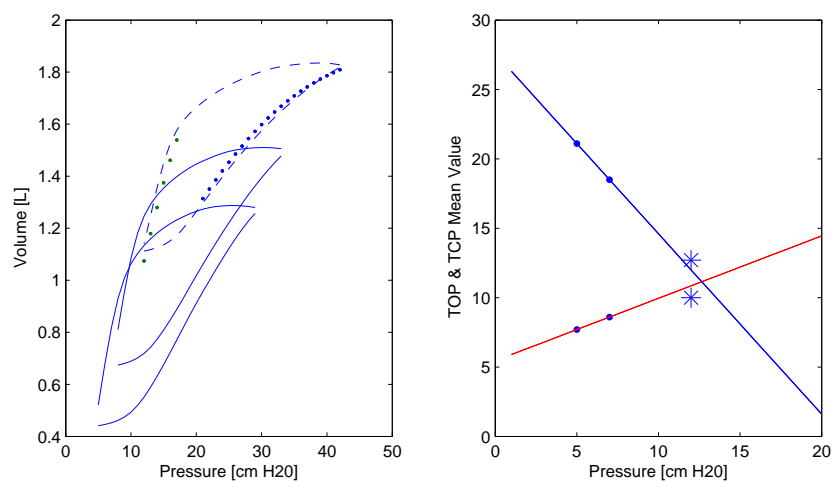

Figure 13: A prediction result for PEEP $=12 \mathrm{cmH}_{2} \mathrm{O}$ of Dataset 1.

this therapy. Once a previously collapsed unit is recruited, it is easier to re-recruit a second time.

Once the unit is initally recruited, the unit can be re-recruited in subsequent cycles at the same PEEP at a lower pressure, essentially decreasing its unit TOP value it had at a lower PEEP. Thus, the overall result is volume increase over the breathing cycle and at a given pressure. 

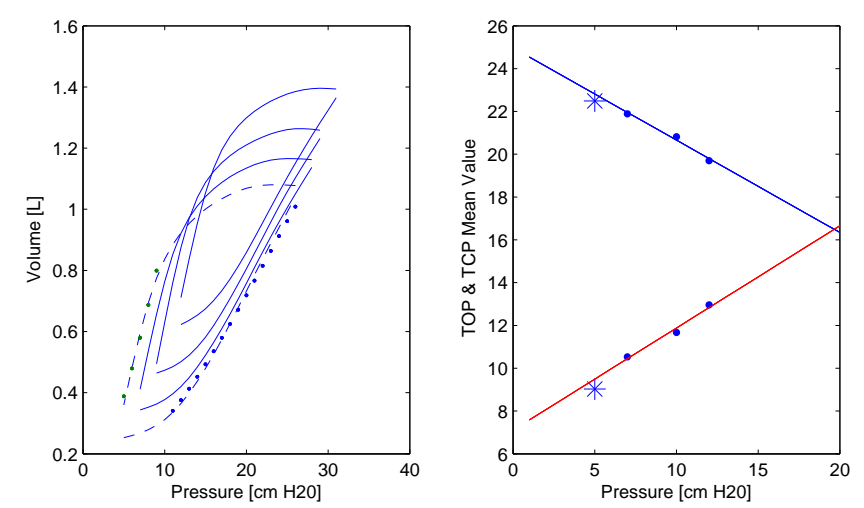

Figure 14: A prediction result for PEEP $=5 \mathrm{cmH}_{2} \mathrm{O}$ of Dataset 5.
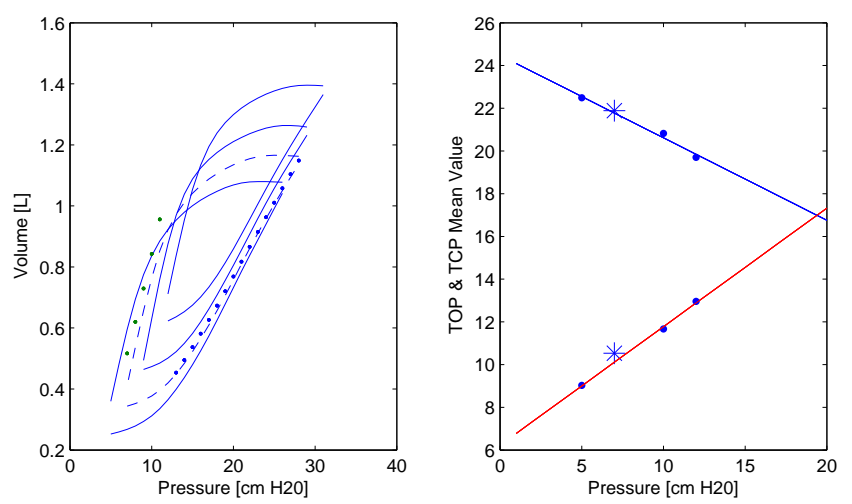

Figure 15: A prediction result for PEEP $=7 \mathrm{cmH}_{2} \mathrm{O}$ of Dataset 5 .

Since the model identifies essential parameters representing lung recruitment, it can be used to track patient recruitment and oxygenation, while the patient is on the ventilator. Thus, when the patient's condition improves or worsens, these parameters change accordingly. By tracking the changes in parameter values over time, the pulmonary condition of the patient can also be tracked. This approach can be used to aid clinical decisions and evaluate the effect of treatment. 

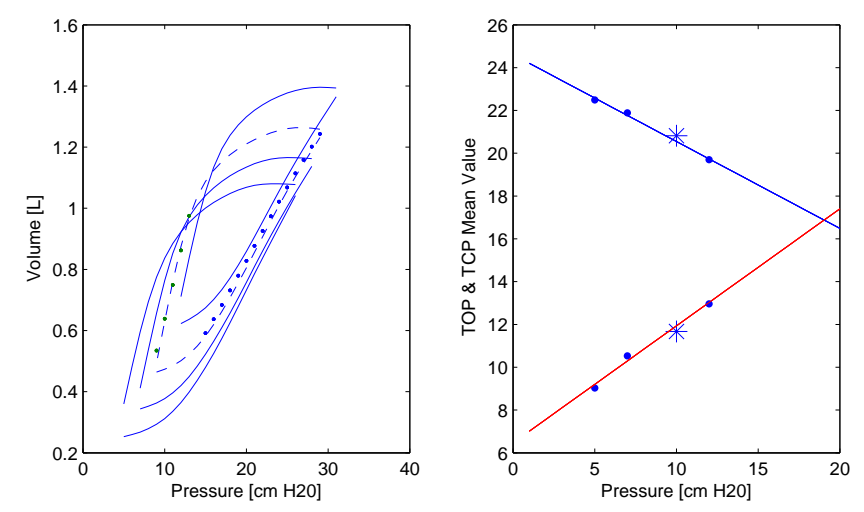

Figure 16: A prediction result for PEEP $=10 \mathrm{cmH}_{2} \mathrm{O}$ of Dataset 5 .
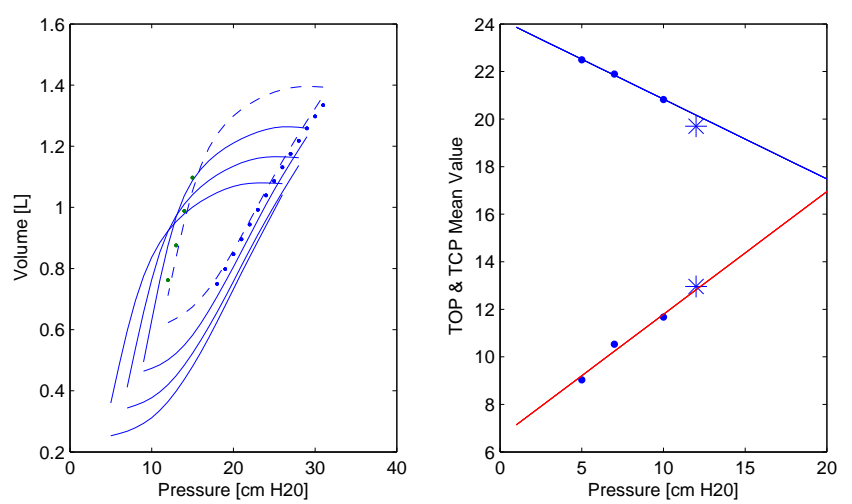

Figure 17: A prediction result for PEEP $=12 \mathrm{cmH}_{2} \mathrm{O}$ of Dataset 5 .

\section{$5 \quad$ Model and Study Limitations}

Currently, the fitting method requires data with deflation to FRC. This data is not typically obtained during current protocols for ventilator treatment. However, this measurement can be obtained by deflating the lung to atmospheric pressure. Once the airway is opened to atmospheric pressure, the lung assumes FRC rapidly, and the entire measuring process can 
be completed in a matter of seconds [31]. This value might have to be obtained one per every 1 - 2 days.

This process requires intervention from the ICU staff and interruption of the patient's breathing pattern, both of which may be clinically unavailable or undesirable. Furthermore, a typical ventilator is only designed to record data on tidal ventilation, so that recording the deflation to FRC would require either a specialised ventilator or a separate data acquisition system. In this cases proposed, clinical model validation will employ external sensor systems.

Similarly, the mean shift trend and prediction of PV loops are primarily based on the relative change of the PV loops with respect to the first PV loop. In other words, for PV predictive purposes, the initial volume or FRC is essentially arbitrary and could be set to 0 . All that is required is the $\triangle \mathrm{FRC}$ after each PEEP intervention. This $\triangle \mathrm{FRC}$ can be easily obtained by a pressure hold at each required increasing PEEP. For example, the change in volume occurring from increasing PEEP from 0 to $5 \mathrm{cmH}_{2} \mathrm{O}$ in a pressure hold maneuver is precisely $\Delta$ FRC. This process could even be integrated into the ventilator to measure $\Delta$ FRC automatically [34].

The mechanics of the lung at the missing PEEP values are readily predicted by linearly fitting the mean shift to identify the impact of this change. It also allows the simplest method for predicting the mean at a new PEEP level. However, some of the means are not perfectly fitted by the linear fit as best illustrated in Figure 13, where the dots are not exactly on the predictive linear line for this relatively extreme PEEP setting. This inaccuracy causes the relatively larger error of the predicted PV curve. Importantly, this error is still within clinical expectations. 
The prediction method utilises just a single parameter TOP (meanshift) to predict between different PEEP settings. Thus, it is simple and easy to use, and can predict an important patient specific response to the change in primary therapy of PEEP. However, it limits other ventilator settings. Since the model parameters were identified using PV loops, only the mechanisms that produced the PV loops are captured. Thus, this prediction method cannot be used to predict changes due to drastically different ventilator settings, such as ventilator mode, flow pattern, maximum inspiratory flow, etc.

However, it should be possible to predict the effect of different tidal volumes, since the EEV is only correlated to PEEP, as long as the flow rates do not change drastically. This impact of flow rates is distinct due to the use of easily available proximal data [35, 36], and the airway resistance changes when flow rates change drastically. This limitation also implies that when the ventilator setting is changed drastically, the model needs to be refit and re-identified using 2 or more PV loops at these new ventilation setting.

That all said, such drastic changes are not typically part of standard ventilator therapy, which focuses more on gradual evolution of settings [37]. In addition, more detailed models could potentially manage such changes, but at a cost of much greater data requirements to create clinically useful patient specific models. Hence, it might be best noted that the model is limited to evolutionary prediction and changes typical of critical care.

This model has the ability to fit and follow the trend of any data including those from a ventilator. Thus, it can reproduce the shape and values of the particular data. For example, if the model was used to identify the parameters for ventilator data, then the model can predict the ventilator data at different settings. Similarly, if carina measurements like those 
of Karason et al [35] are used, then the model can be used to predict the PV curve at the carina for different settings. Thus, the model can be very generally applied to any data set or type that may be available.

It may also be possible to use this model to assess the true lung mechanics, if the ET tube and proximal airway resistances were better known or estimated empirically at the bedside. This approach would require a smarter, more automated ventilator and/or excessive clinical time. However, such smarter ventilators are being developed [34], and may appear in future.

The patient specific mean shift parameter identified by this model is a direct result of analysing raw proximal PV data, which includes dynamic and resistive effects. Therefore, the parameter identified may not directly represent the true lung mechanism. However, because the model is based on the fundamental mechanics, the slope of the mean shift and its clinical relevance can be readily related to the true lung mechanics. One such method to show this relation is to use the estimated carina measurement, which require measurements of the air flow rates and fluid dynamics based estimates of intubation tube resistance, and integrate this aspect in the model.

The model has been validated with limited clinical data from the literature. It therefore requires clinical further validation using more extensive clinical data. The identification methods also need to be validated using additional clinical data. Such data would also enable more direct study of clinical application and patient outcomes.

Finally, more features may need to be included in the model to be fully clinically practical. Such features could include automated data acquisition, parameter identification, airway resistance estimates, and more efficient analysis. However, the overall goal of a clinically 
useful minimally complex model has been proven in concept.

\section{Conclusions}

A minimal model of mechanics of ventilated lung is developed. It employs only 2 unique parameters for each limb of the breathing cycle. Its physiological representation is discussed and compared to more complex not uniquely identifiable physiological model. The model was validated by fitting to clinical data, showing its ability to capture the fundamental mechanics of ventilated lung with reasonable error. Methods for determining different ventilator settings using this model are presented and discussed. The model and its applications are proven in concept and show significant potential as a non-invasive decision-support tool at bedside and could provide non-subjective guidance for optimal ventilation management and continuous patient monitoring.

\section{References}

[1] A. Esteban, A. Anzueto, F. Frutos, I. Alia, L. Brochard, T. E. Stewart, S. Benito, S. K. Epstein, C. Apezteguia, P. Nightingale, A. C. Arroliga, and M. J. Tobin. Characteristics and outcomes in adult patients receiving mechanical ventilation: a 28-day international study. Jama, 287(3):345-55., 2002.

[2] O. R. Luhr, K. Antonsen, M. Karlsson, S. Aardal, A. Thorsteinsson, C. G. Frostell, and J. Bonde. Incidence and mortality after acute respiratory failure and acute respiratory distress syndrome in sweden, denmark, and iceland. the arf study group. Am J Respir Crit Care Med, 159(6):1849-61., 1999. 
[3] T. S. Walsh, S. Dodds, and F. McArdle. Evaluation of simple criteria to predict successful weaning from mechanical ventilation in intensive care patients. $\mathrm{Br} J$ Anaesth, 92(6):793-9. Epub 2004 Apr 30., 2004.

[4] A. D. Bersten, C. Edibam, T. Hunt, and J. Moran. Incidence and mortality of acute lung injury and the acute respiratory distress syndrome in three australian states. $A m$ J Respir Crit Care Med, 165(4):443-8., 2002.

[5] L. B. Ware and M. A. Matthay. The acute respiratory distress syndrome. $N$ Engl $J$ Med, 342(18):1334-49., 2000.

[6] The Acute Respiratory Distress Syndrome Network. Ventilation with lower tidal volumes as compared with traditional tidal volumes for acute lung injury and the acute respiratory distress syndrome. $N$ Engl J Med, 342(18):1301-8., 2000.

[7] M. B. Amato, C. S. Barbas, D. M. Medeiros, R. B. Magaldi, G. P. Schettino, G. LorenziFilho, R. A. Kairalla, D. Deheinzelin, C. Munoz, R. Oliveira, T. Y. Takagaki, and C. R. Carvalho. Effect of a protective-ventilation strategy on mortality in the acute respiratory distress syndrome. N Engl J Med, 338(6):347-54, 1998.

[8] J. M. Halter, J. M. Steinberg, H. J. Schiller, M. DaSilva, L. A. Gatto, S. Landas, and G. F. Niemann. Positive end-expiratory pressure after a recruitment maneuver prevents both alveolar collapse and recruitment/derecruitment. Am J Respir Crit Care Med, 167(12):1620-6. Epub 2003 Feb 25., 2003.

[9] U. G. McCann 2nd, H. J. Schiller, D. E. Carney, L. A. Gatto, J. M. Steinberg, and G. F. Niemann. Visual validation of the mechanical stabilizing effects of positive endexpiratory pressure at the alveolar level. J Surg Res, 99(2):335-42., 2001.

[10] N. D. Ferguson, F. Frutos-Vivar, A. Esteban, A. Anzueto, I. Alia, R. G. Brower, T. E. Stewart, C. Apezteguia, M. Gonzalez, L. Soto, F. Abroug, and L. Brochard. Airway pressures, tidal volumes, and mortality in patients with acute respiratory distress syndrome. Crit Care Med, 33(1):21-30., 2005.

[11] Toshinori Yuta, J. Geoffrey Chase, Geoffrey M. Shaw, and Chirstopher E. Hann. Dynamic models of ards lung mechanics for optimal patient ventilation. In 26th Annual International Conference of the IEEE Engineering in Medicine and Biology Society, San Francisco, CA, 2004.

[12] Toshinori Yuta. Minimal Model of Lung Mechanics for Optimising Ventilator Therapy in Critical Care. PhD thesis, 2007. 
[13] K. G. Hickling. The pressure-volume curve is greatly modified by recruitment. a mathematical model of ards lungs. Am J Respir Crit Care Med, 158(1):194-202, 1998.

[14] K. G. Hickling. Best compliance during a decremental, but not incremental, positive end-expiratory pressure trial is related to open-lung positive end-expiratory pressure: a mathematical model of acute respiratory distress syndrome lungs. Am J Respir Crit Care Med, 163(1):69-78, 2001.

[15] D. E. Carney, C. E. Bredenberg, H. J. Schiller, A. L. Picone, U. G. McCann, L. A. Gatto, G. Bailey, M. Fillinger, and G. F. Niemann. The mechanism of lung volume change during mechanical ventilation. Am J Respir Crit Care Med, 160(5):1697-1702, 1999.

[16] K. G. Hickling. Reinterpreting the pressure-volume curve in patients with acute respiratory distress syndrome. Curr Opin Crit Care, 8(1):32-8., 2002.

[17] H. J. Schiller, J. Steinberg, J. Halter, U. McCann, M. DaSilva, L. A. Gatto, D. Carney, and G. Niemann. Alveolar inflation during generation of a quasi-static pressure/volume curve in the acutely injured lung. Crit Care Med, 31(4):1126-33, 2003.

[18] J. H. Bates and C. G. Irvin. Time dependence of recruitment and derecruitment in the lung: a theoretical model. J Appl Physiol, 93(2):705-13, 2002.

[19] W. Cheng, D. S. DeLong, G. N. Franz, E. L. Petsonk, and D. G. Frazer. Contribution of opening and closing of lung units to lung hysteresis. Respir Physiol, 102(2-3):205-15, 1995.

[20] S. Crotti, D. Mascheroni, P. Caironi, P. Pelosi, G. Ronzoni, M. Mondino, J. J. Marini, and L. Gattinoni. Recruitment and derecruitment during acute respiratory failure: a clinical study. Am J Respir Crit Care Med, 164(1):131-40, 2001.

[21] S. M. Maggiore, B. Jonson, J. C. Richard, S. Jaber, F. Lemaire, and L. Brochard. Alveolar derecruitment at decremental positive end-expiratory pressure levels in acute lung injury: comparison with the lower inflection point, oxygenation, and compliance. Am J Respir Crit Care Med, 164(5):795-801, 2001.

[22] L. M. Malbouisson, J. C. Muller, J. M. Constantin, Q. Lu, L. Puybasset, and J. J. Rouby. Computed tomography assessment of positive end-expiratory pressure-induced alveolar recruitment in patients with acute respiratory distress syndrome. Am J Respir Crit Care Med, 163(6):1444-50., 2001. 
[23] P. Neumann, J. E. Berglund, E. F. Mondejar, A. Magnusson, and G. Hedenstierna. Effect of different pressure levels on the dynamics of lung collapse and recruitment in oleic-acid-induced lung injury. Am J Respir Crit Care Med, 158(5 Pt 1):1636-43., 1998.

[24] P. Pelosi, M. Goldner, A. McKibben, A. Adams, G. Eccher, P. Caironi, S. Losappio, L. Gattinoni, and J. J. Marini. Recruitment and derecruitment during acute respiratory failure: an experimental study. Am J Respir Crit Care Med, 164(1):122-30., 2001.

[25] J. C. Richard, S. M. Maggiore, B. Jonson, J. Mancebo, F. Lemaire, and L. Brochard. Influence of tidal volume on alveolar recruitment. respective role of peep and a recruitment maneuver. Am J Respir Crit Care Med, 163(7):1609-13, 2001.

[26] A. Vieillard-Baron, S. Prin, K. Chergui, B. Page, A. Beauchet, and F. Jardin. Early patterns of static pressure-volume loops in ards and their relations with peep-induced recruitment. Intensive Care Med, 29(11):1929-35, 2003.

[27] G. M. Albaiceta, F. Taboada, D. Parra, A. Blanco, D. Escudero, and J. Otero. Differences in the deflation limb of the pressure-volume curves in acute respiratory distress syndrome from pulmonary and extrapulmonary origin. Intensive Care Med, 29(11):1943-9, 2003.

[28] G. M. Albaiceta, F. Taboada, D. Parra, L. H. Luyando, J. Calvo, R. Menendez, and J. Otero. Tomographic study of the inflection points of the pressure-volume curve in acute lung injury. Am J Respir Crit Care Med, 170(10):1066-72. Epub 2004 Aug 18., 2004.

[29] B. Jonson, J. C. Richard, C. Straus, J. Mancebo, F. Lemaire, and L. Brochard. Pressurevolume curves and compliance in acute lung injury: evidence of recruitment above the lower inflection point. Am J Respir Crit Care Med, 159(4 Pt 1):1172-8, 1999.

[30] J. G. Venegas, R. S. Harris, and B. A. Simon. A comprehensive equation for the pulmonary pressure-volume curve. J Appl Physiol, 84(1):389-95., 1998.

[31] A. D. Bersten. Measurement of overinflation by multiple linear regression analysis in patients with acute lung injury. Eur Respir J, 12(3):526-32, 1998.

[32] G. Foti, M. Cereda, M. E. Sparacino, L. De Marchi, F. Villa, and A. Pesenti. Effects of periodic lung recruitment maneuvers on gas exchange and respiratory mechanics in mechanically ventilated acute respiratory distress syndrome (ards) patients. Intensive Care Med, 26(5):501-7, 2000. 
[33] P. Pelosi, P. Cadringher, N. Bottino, M. Panigada, F. Carrieri, E. Riva, A. Lissoni, and L. Gattinoni. Sigh in acute respiratory distress syndrome. Am J Respir Crit Care Med, 159(3):872-80, 1999.

[34] S. E. Rees, S. Kjaergaard, P. Perthorgaard, J. Malczynski, E. Toft, and S. Andreassen. The automatic lung parameter estimator (alpe) system: non-invasive estimation of pulmonary gas exchange parameters in 10-15 minutes. J Clin Monit Comput, 17(1):43-52., 2002.

[35] S. Karason, S. Sondergaard, S. Lundin, J. Wiklund, and O. Stenqvist. Evaluation of pressure/volume loops based on intratracheal pressure measurements during dynamic conditions. Acta Anaesthesiol Scand, 44(5):571-7., 2000.

[36] S. Karason, S. Sondergaard, S. Lundin, J. Wiklund, and O. Stenqvist. Direct tracheal airway pressure measurements are essential for safe and accurate dynamic monitoring of respiratory mechanics. a laboratory study. Acta Anaesthesiol Scand, 45(2):173-9., 2001.

[37] J. J. Rouby, Q. Lu, and I. Goldstein. Selecting the right level of positive end-expiratory pressure in patients with acute respiratory distress syndrome. Am J Respir Crit Care Med, 165(8):1182-6., 2002. 